\title{
LA OPOSICIÓN ENTRE LA RAZÓN INSTRUMENTAL Y LA LÚDICA EN LA PROPUESTA UTÓPICA DE BERNARD SUITS
}

\author{
FRANCISCO JAVIER LÓPEZ FRÍAS \\ Pennsylvania State University, Estados Unidos \\ XAVIER GIMENO MONFORT \\ Universidad de Valencia
}

\begin{abstract}
RESUMEN: En este texto analizamos el estudio que Bernard Suits hace del uso instrumental de la razón en The Grasshopper a través de su utopía. Para ello, analizamos el papel que juega la razón instrumental en la teoría del juego y en la utopía de Suits. Luego, comparamos el uso instrumental de la razón con lo que denominamos «actitud lúdica». Esta última encarna la racionalidad lúdica que los jugadores han de adoptar para jugar al juego y vivir en el mundo utópico de Suits. Para concluir, defendemos que la actitud lúdica ofrece una alternativa a la predominancia del uso instrumental de la razón en nuestra sociedad.
\end{abstract}

PALABRAS CLAVE: juego; sentido de la vida; razón instrumental; actitud lúdica.

\section{The opposition between instrumental and lusory rationality in the utopian proposal of Bernard Suits}

ABSTRACT: In this paper, we explore Bernard Suits' analysis of the instrumental use of reason in the utopia presented in The Grasshopper. To do so, we analyze the role instrumental rationality plays in Suits' theory of games and utopia. Then, we compare the use of instrumental reason to what we call "lusory rationality." The latter embodies the lusory attitude that players have to adopt to play a game and live in Suits' Utopian world. To conclude, we argue that the lusory attitude offers an alternative to the predominance of the instrumental use of reason in our society.

KEY WORDS: games; meaning of life; instrumental rationality; lusory attitude.

1. Bernard Suits y su atíPica PRopuesta utópica

«La utopía está en el horizonte. Yo sé que nunca la alcanzaré. Que si camino diez pasos, se alejará diez pasos. Cuanto más la busque, menos la encontraré porque ella se va alejando, a medida que yo me acerco. La utopía sirve para eso, sirve para caminar».

Fernando Birri, citado por Eduardo Galeano ${ }^{1}$.

1 Singulars, Eduardo Galeano: ¿Para qué sirve la Utopía?, 2011. https://www.youtube. $\mathrm{com} /$ watch?v=GaRpIBj5xho 
La presencia del juego y los deportes no es común en propuestas filosóficoutópicas modernas ${ }^{2}$. Más bien, resulta todo lo contrario, ambas prácticas aparecen habitualmente en distopías o anti-utopías, tal y como se muestra en obras de ficción como Los juegos del hambre y Rollerball. En éstas, el juego y el deporte promueven contra-valores indeseables para el desarrollo adecuado de la sociedad. Además, en aquellos casos en que el juego y el deporte aparecen en mundos utópicos, como en el de Tomás Moro $^{3}$, lo hacen de un modo superficial e instrumentalizado. Los individuos sólo juegan a juegos y practican deportes esporádicamente y en función a la lógica básica de la sociedad: la organización racional del trabajo y la sociedad.

Dos razones principales se han aportado para explicar la ausencia del deporte en las propuestas utópicas modernas ${ }^{4}$ : la promoción de valores anti-utópicos y la trivialidad de su racionalidad lúdica. La primera razón es aportada por Thompson, quien afirma que el fracaso ligado al confrontamiento propio del juego contradice la naturaleza de utopía. Según él, la decepción y sentimientos de inferioridad asociados a la derrota no tienen lugar en el mejor de los mundos posibles. Por otro lado, la lógica lúdica del juego lo aísla y convierte en trivial y secundario respecto al resto de actividades del día a día. Para alcanzar un mundo ideal, como los presentados por Francis Bacon y Moro, el cultivo de la razón instrumental y el trabajo parecen, pues, mucho más esenciales y «serios» que el del espíritu lúdico del juego.

Aunque el juego y el deporte son generalmente obviados o concebidos como negativos por el pensamiento utópico, Bernard Suits, uno de los padres teóricos de la filosofía del deporte, presenta un mundo ideal llamado «Utopía». En él, jugar a juegos se presenta como el ideal de la existencia humana. Dicha actividad, en oposición al cultivo de la razón instrumental, es la piedra angular del mejor de los mundos posibles. En este trabajo, nos proponemos presentar y analizar la propuesta utópica de Suits, la cual rara vez ha sido considerada por los estudiosos de la utopía.

2 Thompson, K., "Sport and Utopia», en: Journal of the Philosophy of Sport (31, n. ${ }^{\circ} 1$, 2004), 60-63. doi:10.1080/00948705.2004.9714649.

3 Moro apenas hace referencia a los juegos en su utopía. Cuando lo hace, éstos son vistos bien como algo negativo, conducente a la miseria, la apuesta de dinero y el robo, o bien como algo superficial y sometido a los fines y objetivos de la sociedad: «Después de cenar pasan una hora de recreo, durante el verano en el jardín, y en las salas de los comedores públicos durante el invierno. Allí se entregan a la música o se entretienen charlando. Los juegos de azar, como los dados, cartas, tan impropios y nefastos ni siquiera los conocen. No obstante, sí practican dos juegos que se parecen bastante al ajedrez: uno es un combate de números, en el que unos números atrapan a otros. En el segundo, virtudes y vicios entablan una cerrada batalla. Este último juego muestra a las claras la anarquía de los vicios entre sí, y su perfecto acuerdo cuando se trata de luchar contra las virtudes. Hace ver, además, cuáles son los vicios opuestos a determinadas virtudes, qué armas despliegan los vicios cuando atacan por el flanco, qué tropas lanzan a la lucha abierta, y qué posición defensiva permite a las virtudes contener a los ejércitos del vicio, y con qué artimañas burlan sus ataques. Finalmente, hacen ver cuáles son los medios que permiten a uno y otro campo asegurar la victoria». Tomás Moro, Utopía, Tecnos, Madrid, 1987, libro 2.

4 Holowснак, M. A., "Games as Pastimes in Suits's Utopia: Meaningful Living and the "Metaphysics of Leisure" ", en: Journal of the Philosophy of Sport (34, n. ${ }^{\circ}$ 1, 2007), 88-96; HolowCHAK, M. A., y BarKasI, M., "An Impromptu Visit to Rien-à-Faire A Tribute to Bernard Suits», en: Journal of the Philosophy of Sport (35, n. $\left.{ }^{\circ} 2,2008\right), 111-19$. 


\section{SUITS y THE GRASSHOPPER, UN LIBRO TAN CONTROVERTIDO COMO FUNDACIONAL}

The Grasshopper: Games, Life and Utopia (1978), el libro que contiene la propuesta utópica de Suits, es una obra controvertida tanto por su contenido, como por su estructura y características formales ${ }^{5}$. En lo que respecta a la estructura, lo más llamativo es que The Grasshopper constituye un juego en sí mismo ${ }^{6}$. La obra es tanto un ejercicio lúdico para Suits, quien decidió escribirla al modo de un diálogo platónico ${ }^{78}$, como para el lector, quien se encuentra con que el libro concluye con un acertijo sin solucionar que debe resolverse usando la definición de juego presentada por Suits.

En lo que respecta al contenido, la obra se inspira en la «Fábula de la Hormiga y la Cigarra» de Esopo de la que toma el nombre. Suits formula la moraleja de la fábula a la inversa. En vez de mostrar la superioridad del modo de vida de la hormiga, preocupada por las necesidades de la vida diaria, defiende el tipo de vida lúdico de la cigarra. Así pues, como veremos, Utopía es un mundo habitado por cigarras ya que las hormigas, a través de la adopción de lo que Suits denomina como «actitud lúdica» (lusory attitude) ${ }^{9}$, terminan por convertirse en cigarras. Esta inversión de la fábula de Esopo propone un cambio de la racionalidad que, según nuestra interpretación, debe guiar la persecución de un mundo mejor en detrimento de la racionalidad técnico-instrumental.

La propuesta utópica de Suits se presenta justo al final del libro, y tiene la finalidad de ser la pista definitiva que el lector necesita para dar respuesta a los acertijos que la cigarra anuncia a las hormigas en su lecho de muerte ${ }^{10}$. Los acertijos, para complicar más la cuestión, están contenidos en un sueño que acecha

5 McLaughuin, D. W., Reinventing the Wheel: On Games and the Good Life, Tesis de Doctorado dirigida por R. Scott Kretchmar, Pennsylvania State University, 2008.

6 Kolers, A., "The Grasshopper's Error: Or, On How Life is a Game», en: Dialogue: Canadian Philosophical Review/Revue canadienne de philosophie (54, n. ${ }^{\circ}$ 4, 2015), 727-746. doi:10.1017/S0012217315000499.; Suits, B., «Words On Play», en: Journal of the Philosophy of Sport (4, n. ${ }^{\circ} 1$ 1, 1977), 117-31. doi:10.1080/00948705.1977.10654132; Surts, B., «Is Life a Game We Are Playing?», en: Ethics 77, n. 3 (1967): 209-13; Vossen, D., Vossen, D. P., «Utopia is Intelligible and Game-Playing is What Makes Utopia Intelligible», en: Journal of the Philosophy of Sport (43, vol. 2, 2016), 251-265.

7 El hecho de escribir en forma de diálogo debe entenderse como un obstáculo que Suits se autoimpone voluntariamente para formular sus argumentos. Este autoimponerse obstáculos innecesarios, como veremos más adelante, compone la base de su definición del jugar a juegos.

8 López Frías, F. J., y Gimeno Monfort, X., «A Hermeneutical Analysis of the Internalist Approach in the Philosophy of Sport», en: Physical Culture and Sport. Studies and Research (67, n. $\left.{ }^{\circ} 1,2015\right), 5-12$. doi:10.1515/pcssr-2015-0018.

9 El término «lusory» proviene del término latino ludere, el cual tiene que ver con el jugar, con hacer algo con alegría, sólo con el fin de entretenerse o divertirse. Otra posibilidad para traducir esta expresión sería la de «actitud recreativa» o «racionalidad recreativa». La palabra «recreo» se deriva del verbo «recrear» o «recrearse». Este verbo viene del latín recreare, que aparte de significar crear de nuevo, significaba hacer revivir, restablecer, reanimar, reparar o vivificar los ánimos y las fuerzas. Recreo se refiere a aquello que nos vivifica y reanima, porque nos repara del trabajo, nos divierte y nos deleita.

10 Proporcionando una imagen que recuerda a la última reunión de Sócrates con sus discípulos descrita en La apología 
a la cigarra con asiduidad:

«Siempre he tenido, de modo recurrente, un sueño en que se me revela [...] que todas las personas que están vivas están, de hecho, envueltos en jugar a juegos complicados, mientras que al mismo tiempo creen que están ocupándose de sus labores diarias. Los carpinteros, creyendo que están realizado su oficio, están realmente jugando a un juego, y lo mismo sucede con políticos, filósofos, amantes, asesinos, ladrones, y santos. Cualquier ocupación o actividad que puedas concebir, es, en realidad, un juego. Esta revelación es, por supuesto, asombrosa. La consecuencia es terrible. Ya que, en el sueño, luego, me dedico a persuadir a todo el mundo que me encuentro de la gran verdad que me ha sido relevada. Cómo soy capaz de persuadirlos, no lo sé, pero lo hago. Pero en el preciso momento que cada uno es convencido-y esta es la parte terrible—éste deja de existir» ${ }^{11}$.

De entre las adivinanzas planteadas en el sueño, la central es: ¿por qué se desvanece la gente que, pensando que están embarcados en actividades vitales y serias, descubre que, en realidad, sólo están jugando a juegos? Otras adivinanzas son: ¿por qué la gente estaría jugando a juegos? ${ }^{12}$ ¿Qué tipo de juegos? ${ }^{13}$ ¿No sería una vida de jugar a juegos, más bien, una distopía donde la gente se dedica a lo trivial y carente de sentido ${ }^{14}$ Dado que la cigarra fallece tras narrar su sueño, tanto sus discípulos — Prudence y Skepticus — ${ }^{15}$, como el lector, quedan invitados a resolver los acertijos que plantea ${ }^{16}$.

Dado que Prudence y Skepticus fracasan en su intento, el Grasshopper revive y, con el fin de ayudar, ofrece más detalles para la correcta interpretación de su sueño. No obstante, siguiendo con el espíritu lúdico de la obra, no desvela directamente qué significa el sueño ni cómo interpretarlo correctamente, sino que presenta otro acertijo a interpretar (dejando al lector con la tarea de resolver el acertijo): la descripción de una utopía.

Utopía es un mundo en que la razón instrumental ha logrado su realización plena: nadie ha de preocuparse por realizar la más mínima e insignificante tarea ${ }^{17}$,

11 Surts, B., The Grasshopper: Games, Life and Utopia. Peterborough, Ont.: Broadview Press, 2005, 11-12.

12 BäcK, «The Paper World of Bernard Suits», 157.

13 McLaughlin, o.c.; Suits, B., «Games and Utopia Posthumous Reflections», en: Simulation \& Gaming (15, n. $\left.{ }^{\circ} 1,1984\right), 5-24$.

14 Morgan, W. J., "On Suits On the Moral Normativity of Games», University of Toronto, 2015. https://www.humanities.utoronto.ca/uploaded_files/content/1630/file/Morgan_On_ Suits_on_Normativity_of_Games.pdf.

15 La selección de estos nombres para los discípulos a los que la cigarra trata de convertir al modo lúdico de ver el mundo no es casual. Prudence hace referencia a la idea de que solemos afrontar la vida de un modo prudencial, orientando todos nuestros actos a la supervivencia a través de la satisfacción de nuestras necesidades. De este modo, la vida dedicada al trabajo parece mucho más adecuada que la «imprudente» vida dedicada al juego que propone la cigarra. En el caso de Skepticus, su nombre hace referencia a la concepción del juego como algo trivial que no tiene un valor ulterior más allá de la diversión momentánea que nos proporciona. Por el contrario, el trabajo sí permite alcanzar bienes y fines relevantes para nuestra vida. Esta oposición levanta mucho escepticismo en lo que respecta a dotar al juego de un lugar no sólo importante, sino central en nuestras vidas.

16 Kolers, A., o. c., 170.

17 Suits, B., o. c., 2005, 182. 
ya que las máquinas lo hacen todo ${ }^{18}$. En Utopía se ha logrado un estado de máxima eficiencia técnica a la hora de satisfacer los deseos y necesidades biológicas humanas. Además, no hay ningún tipo de competición por amor, atención y aprobación social o admiración. Todo tipo de esfuerzo por satisfacer necesidades sociales es también innecesario. En definitiva, nadie ha de trabajar o esforzarse en Utopía porque la razón instrumental ha sido llevada a su máxima realización, liberando a los sujetos de las imposiciones del mundo de la necesidad. Todo el tiempo a disposición de los habitantes de Utopía es tiempo libre (de trabajo y esfuerzo).

Según Suits, en esta situación, a los individuos de Utopía sólo les quedan dos posibilidades: (a) no hacer nada y, por lo tanto, acabar sumidos en el tedio absoluto, o (b) dedicarse a actividades autotélicas que desean realizar solo por el valor que encierran en sí mismas (en oposición a aquellas que se realizan como medio para satisfacer necesidades y deseos). La decisión de qué camino elegir dependerá del tipo de racionalidad adoptada por cada uno. Si se piensa con la mentalidad de la hormiga, es decir, tratando de maximizar la utilidad de los medios para alcanzar ciertos fines, entonces el tedio será el devenir. Si, por el contrario, el espíritu de hormiga se abandona en favor de la razón, o actitud lúdica de la cigarra, los sujetos acabarán dedicándose a actividades elegidas o autoimpuestas por resultar intrínsecamente valiosas.

\section{Utopía COMO concepto Filosófico. Su SENTIDo Genuino}

Desde un punto de vista etimológico, el concepto «utopía» es un neologismo acuñado y creado por Moro basado en la palabra que los griegos empleaban para referirse a lugar: «topos». Si bien el prefijo «u» no existe como tal en el lenguaje griego, los estudiosos de la obra de Moro coinciden en que la grafía-prefijo «u» proviene del griego «ou» (negación) y «eu» (lo deseable, lo bueno). Partiendo de este análisis etimológico, parece que Moro juega con los prefijos «ou»-topos (no-lugar) y, por otro lado, «eu»-topos (el lugar-deseable o el lugar-bueno) para plasmar el doble carácter de su utopía ${ }^{19}$. El propósito original de la utopía no pasa por presentar un proyecto irrealizable o imposible sino, más bien, uno no-realizado, aún por realizar y, a la sazón, deseablemente realizable. Las utopías no pretenden proponer un mundo idílico o imposible de realizar, sino servir de método o herramienta para criticar la realidad. Las utopías parten de la realidad para volverse contra ella de modo crítico-positivo.

La propuesta utópica de Suits, al igual que la de Moro, es empleada como una herramienta crítica ${ }^{20}$. Para aquellos que interpretan a Suits únicamente como

18 Todo individuo puede tener todo cuanto desee en lo que respecta a las necesidades más básicas: comida, descanso, felicidad, refugio etc. Con sólo pensar algo que necesita o desea, las máquinas se pondrán manos a la obra para proporcionárselo.

${ }_{19}$ Claeys, G., The Cambridge Companion to Utopian Literature, Cambridge University Press, Cambridge; New York, 2010.

20 HolowchaK, M. A., o. c.; Kretchmar, R. S., «The Intelligibility of Suits's Utopia: The View From Anthropological Philosophy», Journal of the Philosophy of Sport (33, n. ${ }^{\circ}$ 1, 2006), 67-77. doi:10.1080/00948705.2006.9714691. 
un teórico del juego, esta herramienta crítica tiene que ver con el jugar a juegos. Es decir, con aquello que hace posible dicha actividad, así como que pueda ser llevada a cabo del mejor modo posible ${ }^{21}$. Por otra parte, aquellos que conciben la intención principal de la obra de Suits más ampliamente, esto es, como ligada a la búsqueda de sentido a la vida, Utopía se revela como la realización del mejor modo de afrontarla. En definitiva, una suerte de interpretación de orden ontológicoantropológico.

Aquí optamos por la segunda opción (aunque sin descartar la primera). Utopía gira en torno al mejor modo de afrontar la vida y, como toda creación utópica, cumple una doble función. Por un lado, identifica la condición (o condiciones) de posibilidad del tipo de racionalidades que el ser humano puede adoptar a la hora de vivir, a saber, la instrumental y la lúdica. Por otro lado, Utopía supone un horizonte de sentido crítico-ideal construido en torno a la actitud más adecuada, a saber, la lúdica de la cigarra. En lo que sigue, procederemos al análisis de cada una de estas funciones.

\subsection{Reconstrucción de las condiciones de posibilidad de Utopía}

La actividad central y condición de posibilidad de Utopía es el jugar a juegos. Esta actividad ejemplifica, según Suits, la razón lúdica. La propuesta utópica de Suits, con el fin de concentrarse en dicha actitud o razón lúdica y convertirse en una herramienta para investigarla, propone un mundo en que el trabajo y actividad instrumental han quedado abolidas. De ese modo, el hombre sólo puede participar en actividades lúdicas, es decir, jugar a juegos. Si esto es así, cabe preguntarse: ¿Por qué es la razón lúdica opuesta a las actividades instrumentales? Para dar respuesta a esta cuestión es necesario recurrir a la definición de jugar a juegos de Suits.

Suits define el jugar a juegos, primero, de un modo breve como «un intento voluntario de superar obstáculos innecesarios» ${ }^{22}$. Luego, en un esfuerzo por completarla y hacerla más comprensible añade ${ }^{23}$ :

«Jugar a un juego es participar en una actividad enfocada a conseguir una determinada situación (meta pre-lúdica), usando sólo los medios permitidos por las reglas (medios lúdicos), en la que las reglas prohíben el uso de los medios más eficientes en favor de los menos eficientes (reglas constitutivas), y en el que las reglas son aceptadas sólo porque hacen posible tal actividad (actitud lúdica)» ${ }^{24}$.

En la concepción de Suits, el juego está constituido por una meta, situación o, si lo traducimos literalmente, «estado de cosas» que se trata de conseguir. Ésta determina el objetivo básico y principal del juego. Por ejemplo, jugar al golf se trata de introducir una pelota dentro de un agujero, en fútbol se busca conducir una pelota con los pies para, colectivamente, hacerla pasar por la línea de anotación

21 LóPEZ FRíAs, F. J., «The reconstructive and normative aspects of Bernard Suit's Utopia», Reason Papers (38, n. ${ }^{\circ}$ 1, 2016), 51-64.

22 Suits, B., o. c., 2005, 43.

23 Hay que remarcar que, aunque la definición se ha empleado de modo indistinto tanto como para referirse a «juego» como a «jugar juegos», Suits parece estar refiriéndose exclusivamente al acto de jugar a juegos (game-playing).

24 Suits, o. c., 2005, 36. 
(línea de gol), en una carrera a pie se compite por llegar de un punto hasta otro siguiendo un circuito determinado. Todos estos ejemplos son muestras del estado de cosas que se trata de conseguir a la hora de jugar a un juego.

Los medios a disposición del participante para lograr la meta propuesta por el juego son múltiples. Por ejemplo, un modo de colocar la pelota en el hoyo de golf es: agarrarla, acercarse al hoyo, e introducir la pelota manualmente. En fútbol se podría, de igual modo, agarrar la pelota y, al modo del rugby, correr hacia adelante para atravesar la línea de gol. En el ejemplo de la carrera a pie, hay diversos modos de llegar desde un punto (A) hasta otro (B), por ejemplo, tomando un taxi, conduciendo un coche, o usando el metro hasta el final del circuito.

En todos estos casos se han empleado medios para lograr un estado de cosas. Sin embargo, no puede decirse que los participantes que han recurrido a dichos medios han jugado al juego del golf, fútbol y corrido una carrera, respectivamente. Ninguno de ellos ha participado de modo adecuado en el juego en cuestión. En algunos casos el medio usado no correspondía al juego en que se pretendía participar, como en el ejemplo del fútbol, mientras que en otros casos no ha jugado a nada, por ejemplo, usando la mano para introducir la pelota de golf en el hoyo.

Para jugar a un juego hace falta dos cosas. Primero, darse cuenta del valor de las normas constitutivas, y, segundo, aceptarlas como limitación de los medios disponibles para alcanzar los fines del juego. El objetivo de las normas es limitar los medios de que disponen los participantes para alcanzar la meta y, con ello, generar un obstáculo o desafío. El sentido y capacidad de atracción del jugar a juegos depende, pues, de la aceptación por parte de los participantes del uso de un número restringido de medios más ineficientes para lograr un objetivo, es decir, de su sometimiento a las reglas constitutivas. La esencia del juego es, pues, las reglas que generan ineficiencia. Así, por ejemplo, en el fútbol, una de sus reglas constitutivas consiste en conducir el balón con cualquier parte del cuerpo menos las manos, siendo los pies los más indicados para ello.

La exclusión de los medios más eficientes (las manos) genera un obstáculo que ha de superarse a través del desarrollo de ciertas «habilidades constitutivas» ${ }^{25}$ que se ponen a prueba constantemente a través del juego. La clave y origen del mismo es, pues, según Suits, la relación que guardan las normas y los fines a través de la cual se seleccionan medios ineficientes para lograr algo que se podría conseguir con el uso de medios más eficientes. Esto muestra que la creación, autoimposición, y aceptación de obstáculos innecesarios para alcanzar la meta deseada es lo que da sentido al jugar a juegos.

La autoimposión de obstáculos innecesarios contrasta con las actitud predominante en el ámbito del trabajo. Suits caracteriza a éste como aquello que "hacemos como medio para otra cosa» ${ }^{26}$. Al trabajar empleamos la razón instrumental para eliminar o minimizar obstáculos y conseguir nuestros fines fácilmente. Este tipo de racionalidad no se preocupa tanto de los obstáculos en sí mismos, sino en alcanzar metas del modo más rápido y eficiente posible. La voluntad y aceptación de obstáculos innecesarios sería vista como absurda en

25 Torres, C. R., "What Counts As Part of a Game? A Look at Skills», Journal of the Philosophy of Sport (27, n. ${ }^{\circ}$ 1, 2000), 81-92. doi:10.1080/00948705.2000.9714591.

26 Suits, B., o. c., 2005, 16. 
actividades como: presentarse a unas elecciones, construir una casa, escribir un cheque, o incorporarse al trabajo cada mañana. En el juego, por el contrario, la clave es, precisamente, la limitación de los medios más efectivos, sólo porque la inneficiencia permite crear el mundo artificial en que el juego es posible ${ }^{27}$.

La disposición de aceptar la limitación de medios que impone las normas, sólo para que el juego sea posible, es lo que Suits denomina «actitud lúdica». Ésta constituye el «pegamento» que mantiene juntos todos los elementos de la definición del jugar a juegos, haciéndolos formar un todo coherente y dotándolos de sentido. De hecho, puede afirmarse que la actitud lúdica es previa a cualquier creación de normas o restricción de medios para imponer obstáculos innecesarios. Si bien las normas del juego son las que indican cómo jugar al juego, los participantes, antes que nada, han de mostrar el deseo y la actitud de querer participar en el mismo limitando, para ello, sus opciones a la hora de conseguir la meta que se revela como lúdica.

La voluntad y deseo de "entregarse a la ineficiencia»-como medio para alcanzar metas determinadas-, configuran las condiciones de posibilidad de lo que hemos llamado anteriormente «razón lúdica». Para completar la descripción de la actitud lúdica, Suits construye su fábula utópica donde los indivíduos, liberados de la necesidad gracias a la máquinas, pueden dedicarse en exclusiva a aquellas actividades auténtica y absolutamente autotélicas, es decir, aquellas que resultan valiosas por sí mismas. En Utopía, esta clase de actividades autotélicas están representadas por el «jugar a juegos». Si esto es así, cualquier actividad que no quede sustentada por el espíritu lúdico, queda fuera de Utopía por ser instrumental.

La voluntad y deseo de «entregarse a la ineficiencia»-como manera de alcanzar una meta determinada- configuran las condiciones de posibilidad de ese modo de afrontar la vida que hemos denominado «razón lúdica». Para completar su descripción de la actitud lúdica, Suits emplea la ficción de Utopía, de la que dicha actitud es un pilar central y condición de posibilidad. En Utopía, los individuos sólo juegan a juegos, entendiendo a éstos como la superación de obstáculos innecesarios para alcanzar un fin. Esta actividad es la única que, según nuestro autor, puede considerarse como auténtica y puramente autotélica y, por lo tanto, la única en la que el ser humano puede participar. Toda actividad en que haya un mínimo grado de instrumentalidad, no puramente autotélica, es realizada por las máquinas absolutamente eficientes (que realizan el sueño de la razón instrumental). De este modo, cualquier actividad que no sea completamente autotélica es excluida de Utopía. A través de la eliminación de actividades con un mínimo de instrumentalidad, Suits centra nuestra atención exclusivamente en el jugar a juegos, que es el elemento sin el que Utopía carece de sentido.

Un ejemplo de prácticas que no tienen lugar en su ficción utópica, por contener grados de instrumentalidad, son las actividades de ocio que comúnmente tomamos como conducentes a la felicidad. Así, por ejemplo, si bien Aristóteles tomó la contemplación como la única ocupación intrínsecamente valiosa, para Suits la actividad intelectual no puede ser concebida como tal porque posee un cierto grado de instrumentalidad. La contemplación persigue un fin ulterior: el conocimiento. En Utopía, como hemos indicado, las personas no participan en actividades por

$27 \quad$ Ibid., 43. 
motivos externos con un carácter instrumental, éstas quedan eliminadas por el uso de las máquinas. De este modo, la búsqueda de la verdad por uno mismo carece de sentido, desde el preciso momento en el que las máquinas disponen y pueden proveernos de todo el conocimiento habido y por haber.

Un modo para comprender mejor la distinción que Suits establece entre trabajo, ocio, y juego es dibujar una línea que tiene en un extremo el valor extrínseco y productivo, y en el otro el valor intrínseco (véase figura 1). Mientras que el trabajo se encuentra en el extremo de las actividades de tipo extrínseco, ya que las hacemos como medio para alcanzar un fin externo a la actividad en cuestión, el jugar a juegos representa el valor intrínseco en estado puro. El ocio, por su parte, se encuentra entre ambos, pero más cercano al juego. Actividades de ocio como irse de vacaciones, tocar el trombón, o leer libros, según Suits, se hacen como fines en sí mismos, de modo autotélico, pero siguen teniendo un cierto valor instrumental del que carecen los juegos. El que va de vacaciones quiere visitar lugares del mundo, el que toca un instrumento quiere aprender a dominarlo hasta ser un virtuoso, y, por último, quien lee novelas quiere conocer el contenido y desenlace de las mismas. Estos fines otorgan un carácter instrumental a dichas actividades. El jugar a juegos, según Suits, al menos, concebido en estado puro, trascendental y por qué no decirlo, utópico, carece de este tipo de carácter; el objetivo a conseguir es inconsecuente para la vida diaria, ya que sólo tiene sentido dentro de las barreras artificiales creadas por las normas. El hecho de carecer de objetivos ulteriores convierte al juego en una actividad puramente autotélica, «contenida en sí misma» ${ }^{28}$.

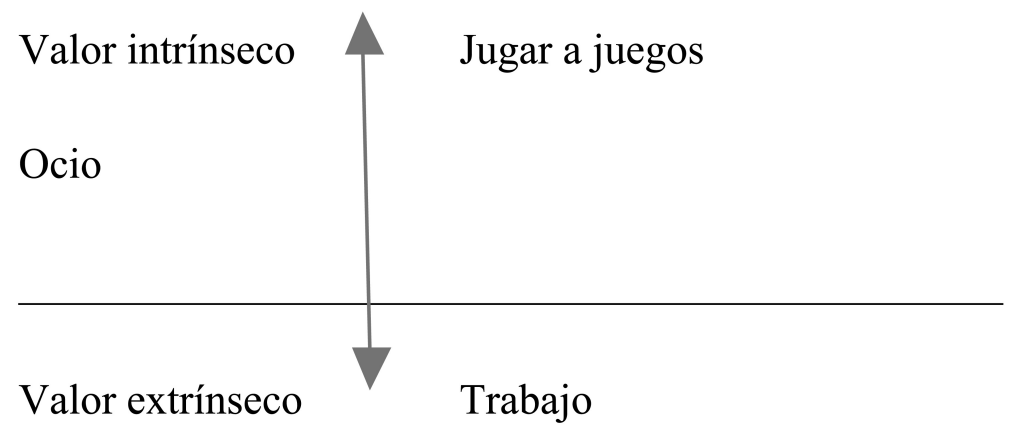

Figura 1

${ }_{28}$ Esto no quiere decir que la racionalidad instrumental quede completamente fuera de la actividad del jugar a juegos. Esta sí tiene un lugar, pero dentro de la esfera delimitada por las reglas artificiales que dan sentido al juego. Así, para Suits, el jugar a juegos es la única actividad en que: «lo instrumental está inseparablemente mezclado con aquello que es intrínsecamente valioso, sin que la actividad sea un instrumento para otro fin. [...] En los juegos tenemos obstáculos por los que luchamos por poder superar sólo porque podemos realizar la actividad como tal, a saber, jugar el juego. Jugar a juegos hace posible mantener el suficiente esfuerzo en Utopía como para que merezca la pena vivir» Surts, B., o. c., 2005, 188-189. 
Profundizando en la distinción entre jugar a juegos y las actividades de ocio, pueden añadirse dos cosas. Primero, el ocio siempre tiene una finalidad extrínseca más allá de la actividad en sí misma. Quienes participan en actividades de ocio se entregan a ellas para ocupar su tiempo. Estas actividades tratan de entretenernos. Segundo, como defiende Kretchmar ${ }^{29}$, el juego tiene una capacidad de atracción intrínseca que no poseen las actividades de ocio. Dado que éstas poseen un fin, una vez que éste se ha conseguido, las actividades pierden sentido. Así, por ejemplo, cuando un viajero ha visitado todos los lugares del mundo, un lector ha leído todas las novelas existentes, o un guitarrista se ha convertido en un virtuoso de su instrumento, estas actividades pierden parte de su valor y atracción. Se tornan monótonas, tediosas, o poco desafiantes. De hecho, parece que el modo de recuperar la capacidad de atracción de estas actividades pasa por convertirlas en un juego imponiendo obstáculos innecesarios. Por ejemplo, el virtuoso de la guitarra decide tocar con su mano menos hábil para convertir dicha actividad en un juego (imponiendo obstáculos innecesarios).

Hay que enfatizar que estos conceptos son teóricos, es decir, referidos a un ámbito transcendental de reflexión, y que, por ello, no pueden encontrarse en el mundo fáctico de un modo completo. Como muestra Aristóteles respecto a las motivaciones para la acción, éstas nunca son puramente intrínsecas o extrínsecas sino mixtas. En un mundo no utópico, cualquier instancia del jugar a juego es de tipo mixto. Lo cual no impide que Suits presente un conjunto de categorías transcendentales que condensan los elementos esenciales de los tres tipos de actividades que le ocupan. Acentuar el carácter transcendental de éstos es, justamente, el objetivo de su Utopía dado que, en definitva, pretende eliminar cualquier tipo de actividades que no casen con las condiciones necesarias y suficientes de su definición de jugar a juegos.

El carácter transcendental de la definición de Suits implica que Utopía no es un lugar dónde los individuos dedican su vida a esas actividades que coloquialmente denominamos como juego, sino proporcionar un método o actitud para que afrontemos la vida de modo lúdico, convirtiendo todas nuestras actividades en juegos que casan con su definición. Es decir, no tiene sentido decir que el día a día de los habitantes de Utopía consiste en correr una carrera por la mañana, jugar al fútbol por la tarde, y a las cartas o la petanca por la noche. La definición del juego de Suits cubre mucho más de lo que habitualmente entendemos como tal, de igual modo que su propuesta utópica va más allá de la comprensión coloquial del término como un mundo perfecto a realizar. Esto puede resultar, como advierte Suits en el prefacio de su obra, "desconcertante» y "polémico», pero ésta es justamente la clave para interpretar filosóficamente tanto su noción de juego, como Utopía.

Como afirma en un artículo posterior a The Grasshopper titulado «Games and Utopia ${ }^{30}$, la vida en Utopía consiste en jugar a juegos complicados y muy elaborados. En lo que sigue, explicaremos a qué tipo de juegos se refiere y cómo ello nos ofrece una comprensión normativa del jugar a juegos como clave para comprender un

29 KREtchmar, R. S., O. c., 2006.

30 Suits, B., o. c., 1984. 
modo lúdico de estar-en-el-mundo-jugando-a-juegos que se corresponde con la razón lúdica.

\subsection{Utopía como horizonte crítico de sentido. El imperativo de la razón lúdica}

El objetivo central de la utopía suitsiana es pensar un mundo donde todos nuestros objetivos y deseos han sido conseguidos. De este modo, no queda ninguna necesidad que satisfacer o deseo pendiente. Es un mundo en el que, como afirma Suits, parece que "no hay nada que hacer o lograr». Un mundo así, no obstante, parece más propio de una anti-utopía o distopía ${ }^{31}$, pues los sujetos caerían en un vacío existencial de aburrimiento absoluto y pasividad. En base a los pronosticos suitsianos, cabe preguntarse: ¿Han de resignarse pues los habitantes de Utopía a encontrar algo que hacer, algún tipo de actividad por el que merezca la pena luchar, que dé sentido a su vida, sin que ésta acabe derivando en una situación de tedio absoluto (entiendo, como sugiere Doug McLaughlin, «tener algo que hacer» como el encontrar sentido vital a las actividades que realizan)? ${ }^{32}$ No, según Suits, a los habitantes de Utopía siempre les queda el jugar a juegos: «El jugar a juegos [, éste es] el único candidato que nos queda para una actividad utópica, y, por lo tanto, el único restante para constituir la existencia ideal» ${ }^{33}$.

Aplicando la definición de Suits, cualquier actividad, modificada como es debido, puede ser convertida en un juego aplicando la razón lúdica. Todo lo que se necesita es restringir los medios a disposición del participante para alcanzar el objetivo de la actividad. Con la restricción de los medios a emplear para su realización, se genera un mundo artificial en que los participantes dotan de prioridad al modo de alcanzar los objetivos, es decir, los medios lúdicos, en vez de a los objetivos como tal. Esta es la característica central de eso que hemos denominado «razón lúdica» que, tal y como venimos advirtiendo, se opone diamentralmente a la razón instrumental dominante en nuestras sociedades.

La clave de guiarnos por la razón lúdica que, precisamente, es el sujeto, al dar prioridad a la ineficiencia y las limitaciones que generan las reglas, se autoimpone a sí mismo los límites y medios adecuados para su acción. De ese modo, los acepta porque tienen un valor intrínseco como parte del juego en que ha decidido participar. Nada le es impuesto desde fuera a los jugadores que habitan Utopía, sino que estos, como afirma Suits: "sólo hacen aquellas cosas que consideran intrínsecamente valiosas [...] siempre hacen cosas porque quieren no porque deban $»^{34}$. La ficción utópica de Suits, por lo tanto, presenta un mundo donde los individuos, guiados por un imperativo lúdico, generan un reino de jugadores que eligen las actividades en que quieren participar sólo en base a razones autotélicas, nada les viene dado por necesidad. Evidentemente, en un mundo no-utópico, la satisfacción de necesidades y deseos de modo instrumental es inevitable. Pero, esto no significa que el imperativo de la razón lúdica característico de Utopía, no pueda

31 Morgan, W. J., " "Some Further Words on Suits on Play"», en: Journal of the Philosophy of Sport (35, n. ${ }^{\circ}$ 2, 2008), 120-41. doi:10.1080/00948705.2008.9714734.

32 HolowchaK, M. A., o. c, 2007.

33 Suits, B., o. c., 2005, 185.

34 Ibid., 191. 
implementarse como horizonte de sentido al que, progresivamente, pudieramos aproximarnos $^{35}$.

Para ilustrar esta cuestión, hemos de atender a los ejemplos que Suits aporta de prácticas habituales tornadas en juegos tras la adopción de la actitud lúdica, a saber: los casos de John Striver (John Luchador) y William Seeker (William Buscador). John Luchador es un habitante de Utopía que se encuentra aburrido, buscando algún tipo de actividad en la que participar. En su afán de hacer algo para dar sentido a su vida, decide que quiere hacer carpintería. Como las máquinas se encargan de proveer todas las casas que la gente de Utopía necesita, no hay ninguna demanda de casas. No obstante, Striver no está interesado en tener una casa, sino en probar y desarrollar sus habilidades como carpintero. Así pues, decide obviar el uso de las máquinas y hacer una casa por sí mismo. Una vivienda que le resulte difícil de construir, pero no imposible ni muy fácil, es decir, algo que suponga un obstáculo desafiante con el que se divierta.

En el caso de William Buscador, la actividad que desea realizar es la persecución de la verdad científica. En su caso, de nuevo, todo el conocimiento posible está disponible, de modo que no hay nada por descubrir. Sin embargo, esto no importa en lo que se refiere a su posibilidad de participar en un juego. La clave reside en la actitud de William para ponerse obstáculos innecesarios y el proceso de superarlos. Para ello, puede decir: "no me digan la respuesta; dejadme alcanzarla por mí mismo» ${ }^{36}$. Así, William rechaza los medios más efectivos de los que dispone para conocer la verdad, y trata de alcanzarla por sí mismo a través de medios menos efectivos.

\section{Para Suits,}

«esta[s] actividad[es] [tanto la carpintería de John y la búsqueda de conocimiento de William], en esencia, no [son] distinta[s] de jugar al golf o a otro juego. De igual modo que, fuera de la práctica del golf, no hay necesidad para poner pelotas en agujeros en el suelo, en Utopía, fuera de la carpintería, no se necesita la casa que va a ser producida en esa actividad de carpintería [...] Lo que es importante para John y el golfista es el acto de lograr esos resultados más que los resultados en sí mismos. Ambos [...] participan en intentos voluntarios de superar obstáculos innecesarios» ${ }^{37}$.

Esto pone en el foco de atención, de nuevo, el hecho de que Utopía gira en torno a la actitud lúdica y trata de, a través de la imaginación contrafáctica, presentar un mundo donde la adopción de la actitud lúdica es el mejor modo posible de afrontar la vida. Con la debida actitud, los habitantes de Utopía convierten todas y cada una

35 De hecho, ésto ha acontecido en aquellas etapas de nuestra historia donde ciertas clases sociales se han convertido en "ociosas» al disponer de privilegios que les permitían no tener que preocuparse por la satisfacción de sus necesidades más básicas, estando desligados del reino de la necesidad que Suits elimina en su utopía. Por ejemplo, los hombres libres de la Grecia antigua pudieron dedicarse a crear los juegos debido a que poseían esclavos que trabajaran para ellos. Otro ejemplo más reciente es la clase aristocrática de la Inglaterra victoriana que, debido a los privilegios y haciendas que poseían, pudieron afrontar la vida y muchas actividades siguiendo el espíritu caballeresco propio del ideal amateur.

36 Suits, o. c., 2005, 191.

37 Ibid., 191-92. 
de sus actividades en juegos en los que su actividad sea intrínsecamente valiosa. No obstante, los «utopianos» tienen otra opción, a saber: dejarse llevar por las máquinas y caer en el vacío existencial del «no tener nada que hacer».

La interpretación antropológica de fondo en la obra de Suits es la visión del ser humano como el animal en busca de sentido. Si, en Utopía, no jugar a juegos lleva al tedio absoluto, sólo el jugar puede salvarnos de caer en el vacío existencial. En nuestras sociedades, extraemos sentido a través de nuestras actividades productivas e instrumentales, y tratamos de expandir el imperio y dominio de la razón instrumental. Éste, sin embargo, no puede ser el modo de dotar de sentido a la vida para el habitante de Utopía, ya que no dispone de la posibilidad de realizar actividades instrumentales. Las hormigas no tienen lugar en Utopía. Esto explicaría que, en el sueño presentado por la cigarra, las hormigas dedicadas al trabajo y la búsqueda de los medios más eficientes se desvanezcan al ser conscientes de que, en Utopía, sólo se juega a juegos y no hay lugar para lo instrumental. Esta es la realidad de Utopía. Para las hormigas, Utopía es una pesadilla. Una distopía que niega su propia esencia, el poder trabajar y embarcarse en actividades de carácter instrumental.

La revelación existencial que traumatiza a la hormiga contiene una lección normativa para nosotros. Primero, porque ya vivimos un mundo colonizado por la razón instrumental. Utopía no ha de pensarse en términos de una sociedad futura fantástica que puede no ser existente. Segundo, la consecución de utopía/ distopía está contenida en la propia esencia de nuestra sociedad, pues el sueño de la razón instrumental consiste, precisamente, en el uso más eficiente de la técnica para doblegar el mundo y tratar de satisfacer nuestros deseos y necesidades. Con ello, Suits indica, de modo normativo, que en nuestra sociedad, dominada por la racionalidad instrumental del homo economicus, el mejor modo de derivar sentido sería afrontar la vida con un carácter lúdico, llenar nuestra vida de juegos con valor autotélico (elegidos por nosotros mismos, no impuestos por el imperio de la necesidad). Así pues, según Suits, hemos de promover y dirigirnos hacia la razón lúdica prestando más atención a las actividades mismas, en vez de a los fines a conseguir a través de ellas.

\section{CONCLUSIÓN: ¡CONVIRTÁmONOS EN CIGARRAs!}

En este artículo hemos analizado la función que posee la ficción utópica creada por Suits en The Grasshopper. Para ello, hemos partido de la concepción filosófica de la utopía, entendida como constructo para: (a) mostrar las condiciones de posibilidad de un fenómeno, y (b) actuar como horizonte de sentido crítico del mismo. Así, hemos defendido que para Suits, Utopía representa una herramienta a través del cual podemos captar de modo esencial cuáles son las condiciones de posibilidad del jugar a juegos, siendo la actitud lúdica el elemento clave; y, además, proporcionar un principio normativo referido al espíritu que adoptamos al jugar a juegos.

Con ello, defendemos que Suits convierte en una cuestión antropológica su reflexión sobre el juego, para tratar de inculcar el espíritu lúdico en todo cuanto 
hacemos. Frente a la racionalidad instrumental del homo economicus, Suits apuesta por el desarrollo e implementación de la racionalidad lúdica del homo ludens como vía de escape a la presión instrumental del mundo actual. En opinión de Suits, la razón instrumental no presta tanta atención al resultado y objetivo de nuestras actividades, como a la actividad en sí misma, es decir, al proceso a través del que las realizamos y el modo en que las hacemos. De este modo, con Utopía, Suits nos invita a convertirnos en cigarras adoptando una actitud lúdica frente a la vida.

\section{BiBLIOGRAFÍA}

Bäck, A. (2008). «The Paper World of Bernard Suits», en: Journal of the Philosophy of Sport (35, n. ${ }^{\circ} 2$, 2008), 156-74. doi:10.1080/00948705.2008.9714736.

Claeys, G. (2010). The Cambridge Companion to Utopian Literature, Cambridge University Press. Cambridge; New York.

Holowchak, M. A. (2007). "Games as Pastimes in Suits's Utopia: Meaningful Living and the "Metaphysics of Leisure" ", en: Journal of the Philosophy of Sport (34, n. ${ }^{\circ}$ 1, 2007), 88-96. doi:10.1080/00948705.2007.9714712.

Holowchak, M. A., y Barkasi, M. (2008). «An Impromptu Visit to Rien-à-Faire A Tribute to Bernard Suits», en: Journal of the Philosophy of Sport (35, n. ${ }^{\circ}$ 2, 2008), 111-19. doi:10.108 0/00948705.2008.9714733.

Kolers, A. (2015). «The Grasshopper's Error: Or, On How Life is a Game», en: Dialogue: Canadian Philosophical Review/Revue canadienne de philosophie (54, n. ${ }^{\circ}$ 4, 2015), 727-746. doi:10.1017/S0012217315000499.

Kretchmar, R. S. (2006). «The Intelligibility of Suits's Utopia: The View From Anthropological Philosophy», Journal of the Philosophy of Sport (33, n. ${ }^{\circ}$ 1, 2006), 67-77. doi:10.1080/0094 8705.2006.9714691.

Lopez Frias, F. J. (2016). «The reconstructive and normative aspects of Bernard Suit's Utopia», Reason Papers (38, n. $\left.{ }^{\circ} 1,2016\right)$, 51-64.

Lopez Frias, F. J., y Gimeno Monfort, X. (2015). «A Hermeneutical Analysis of the Internalist Approach in the Philosophy of Sport», en: Physical Culture and Sport. Studies and Research (67, n. ${ }^{\circ} 1$, 2015), 5-12. doi:10.1515/pcssr-2015-0018.

McLaughlin, D. W. (2008). Reinventing the Wheel: On Games and the Good Life, Tesis de Doctorado dirigida por R. Scott Kretchmar, Pennsylvania State University, 2008.

Morgan, W. J. (2015). «On Suits On the Moral Normativity of Games», University of Toronto, 2015. https://www.humanities.utoronto.ca/uploaded_files/content/1630/file/Morgan_On_ Suits_on_Normativity_of_Games.pdf.

- (2008). "Some Further Words on Suits on Play"», en: Journal of the Philosophy of Sport (35, n. ${ }^{\circ} 2$, 2008), 120-41. doi:10.1080/00948705.2008.9714734.

Singulars (2011). Eduardo Galeano: ¿Para qué sirve la Utopía?, 2011. https://www.youtube. com/watch? $v=$ GaRpIBj5xho.

Suits, B. (1984). «Games and Utopia Posthumous Reflections», en: Simulation \& Gaming (15, n. $\left.{ }^{\circ} 1,1984\right), 5-24$. doi:10.1177/0037550084151002.

— (1967). «Is Life a Game We Are Playing?», en: Ethics 77, n. ${ }^{\circ} 3$ (1967): 209-13.

- (2005). The Grasshopper: Games, Life and Utopia. Peterborough, Ont.: Broadview Press.

- (1977). «Words On Play», en: Journal of the Philosophy of Sport (4, n. ${ }^{\circ}$ 1, 1977), 117-31. do i:10.1080/00948705.1977.10654132.

Thompson, K. (2004). «Sport and Utopia», en: Journal of the Philosophy of Sport (31, n. ${ }^{\circ} 1$, 2004), 60-63. doi:10.1080/00948705.2004.9714649. 
Tomás Moro (1987). Utopía. Madrid: Tecnos.

Torres, C. R. (2000). "What Counts As Part of a Game? A Look at Skills», Journal of the Philosophy of Sport (27, n. ${ }^{\circ}$ 1, 2000), 81-92. doi:10.1080/00948705.2000.9714591.

Vossen, D. P. (2016). "Utopia is Intelligible and Game-Playing is What Makes Utopia Intelligible», en: Journal of the Philosophy of Sport (43, vol. 2, 2016), 251-265. doi:10.108 0/00948705.2015.1112235.

Kinesiology Department y Rock Ethics Institute

Francisco JaVIER LóPEZ Frías Pennsylvania State University, Estados Unidos

Fj113@psu.edu

Universidad de Valencia

xagimon@hotmail.com

Xavier Gimeno Monfort

[Artículo aprobado para publicación en diciembre de 2017] 